\title{
2D Fundamental Solutions for the General Anisotropic Solids with Computer Codes
}

\author{
Mitsunori Denda \\ Rutgers University, Mech. \& Aero. Engng. Dept. \\ Piscataway, NJ 08854-8058, USA, denda@rutgers.edu
}

\begin{abstract}
Basic equations with detailed derivation for the two-dimensional static fundamental solutions are presented for the general anisotropic solids. The displacement, stress and traction solutions for the line force and dislocation fundamental solutions are given along with FORTRAN 90 computer codes.
\end{abstract}

\section{Introduction}

Two-dimensional fundamental solutions for the general anisotropic elastic solids, defined by 21 elastic constants, are derived. The generalized plane strain is assumed. After a short review of Stroh's complex variable formalism for 2D anisotropic elasticity (Stroh [1, 2], Ting [3], Suo [4] and Ni and Nemat-Nasser [5]) the fundamental solutions for the line force and line dislocation are derived following Denda [6] and Denda and Marante [7]. The explicit form of the fundamental solutions are given for the displacement, traction and stress components for the line force and dislocation. Brief description of the downloadable FORTRAN 90 codes is given.

\section{Basic Equations}

For the generalized plane strain anisotropic elasticity problems considered the displacement $\mathbf{u}=\left\{u_{1}, u_{2}, u_{3}\right\}^{T}$ depends only on two coordinates $x_{1}$ and $x_{2}$. This is the case if the geometry and the loading do not vary in the $x_{3}$-axis (i.e., out-ofplane) direction. If the anisotropic material has a symmetry plane parallel to the $x_{1} x_{2}$-plane, then the in-plane $\left(u_{1}\right.$ and $\left.u_{2}\right)$ and the out-of-plane $\left(u_{3}\right)$ deformations become uncoupled. Otherwise, full coupling exists between two deformations. The latter is assumed in this paper including the former as a special case. The equilibrium equation, in the absence of the body force, is given in a vector form by

$$
\frac{\partial \boldsymbol{\sigma}_{1}}{\partial x_{1}}+\frac{\partial \boldsymbol{\sigma}_{2}}{\partial x_{2}}=\mathbf{0}
$$

where $\boldsymbol{\sigma}_{1}=\left\{\sigma_{11}, \sigma_{12}, \sigma_{13}\right\}^{T}$ and $\boldsymbol{\sigma}_{2}=\left\{\sigma_{21}, \sigma_{22}, \sigma_{23}\right\}^{T}$ are the stress vectors. In describing the field variables we replace a pair of suffices $i j$ by a single suffix $M$ following the convention $(11 \rightarrow 1),(22 \rightarrow 2),(33 \rightarrow 3),(23 \rightarrow 4),(31 \rightarrow 5$, $(12 \rightarrow 6)$. The non zero strain components are given by

$$
e_{1}=\frac{\partial u_{1}}{\partial x_{1}}, \quad e_{2}=\frac{\partial u_{2}}{\partial x_{2}}, \quad e_{4}=\frac{\partial u_{3}}{\partial x_{2}}, \quad e_{5}=\frac{\partial u_{3}}{\partial x_{1}}, \quad e_{6}=\frac{\partial u_{2}}{\partial x_{1}}+\frac{\partial u_{1}}{\partial x_{2}}
$$


The strain-stress relations are given by

$$
e_{M}=\sum_{N=1}^{6} S_{M N} \sigma_{N} \quad(\mathrm{M}, \mathrm{N}=1,2,4,5,6),
$$

where $S_{M N}$ is the reduced compliance defined by

$$
S_{M N}=s_{M N}-\left(s_{M 3} s_{3 N}\right) / s_{33} \quad(\mathrm{M}, \mathrm{N}=1,2,4,5,6)
$$

in terms of the compliance $s_{i j}(i, j=1,2,3,4,5,6)$. In this paper no summation convention is used for repeated indices unless mentioned otherwise. The compatibility equations are given by

$$
\frac{\partial^{2} e_{2}}{\partial x_{1}^{2}}+\frac{\partial^{2} e_{1}}{\partial x_{2}^{2}}-\frac{\partial^{2} e_{6}}{\partial x_{1} \partial x_{2}}=0, \quad \frac{\partial e_{4}}{\partial x_{1}}-\frac{\partial e_{5}}{\partial x_{2}}=0 .
$$

If we introduce a real-valued stress function vector $\phi=\left\{\phi_{1}, \phi_{2}, \phi_{3}\right\}^{T}$ such that

$$
\boldsymbol{\sigma}_{1}=-\frac{\partial \phi}{\partial x_{2}}, \quad \boldsymbol{\sigma}_{2}=\frac{\partial \phi}{\partial x_{1}}
$$

then the equilibrium equation (1) is automatically satisfied.

Lekhnitskii [8] and Eshelby et al. [9] have shown that the solution of the generalized plane strain problem can be represented by three functions $f_{1}\left(z_{1}\right)$, $f_{2}\left(z_{2}\right), f_{3}\left(z_{3}\right)$, each of which is analytic in its argument $z_{\alpha}=x_{1}+p_{\alpha} x_{2}$. Here $p_{\alpha}$ are three distinct complex numbers: roots of the sixth-order polynomial characteristic equation (7). The arguments $z_{\alpha}=x_{1}+p_{\alpha} x_{2}(\alpha=1,2,3)$ are called the generalized complex variables. Lekhnitskii [8] has assumed the stress function vector of the form

$$
\phi=1 f\left(x_{1}+p x_{2}\right),
$$

where $\mathbf{l}=\left\{L_{1}, L_{2}, L_{3}\right\}^{T}$, which is substituted in the compatibility equations (5). This results in the sixth-order characteristic equation in $p$

$$
d^{(4)}(p) d^{(2)}(p)-d^{(3)}(p) d^{(3)}(p)=0,
$$

where

$$
\begin{gathered}
d^{(4)}(p)=p^{4} S_{11}-2 p^{3} S_{16}+p^{2}\left(2 S_{12}+S_{66}\right)-2 p S_{26}+S_{22}, \\
d^{(3)}(p)=p^{3} S_{15}-p^{2}\left(S_{14}+S_{56}\right)+p\left(S_{25}+S_{46}\right)-S_{24}, \\
d^{(2)}(p)=p^{2} S_{55}-2 p S_{45}+S_{44} .
\end{gathered}
$$

Lekhnitskii [8] has shown that (7) has no real roots and has three pairs of conjugate complex roots $p_{1}, \bar{p}_{1}, p_{2}, \bar{p}_{2}, p_{3}, \bar{p}_{3}$. The imaginary part of $p_{\alpha}(\alpha=1,2,3)$ is assumed, without loss of generality, positive. It is also assumed that the three roots $p_{1}, p_{2}, p_{3}$ are distinct. In the numerical treatment the coincident roots can be made distinct by slightly perturbing compliance coefficients. The matrix

$$
\mathbf{L}=\left[\mathbf{l}_{\mathbf{1}}, \mathbf{l}_{\mathbf{2}}, \mathbf{l}_{\mathbf{3}},\right]=\left[\begin{array}{ccc}
-p_{1} L_{21} & -p_{2} L_{22} & -p_{3} l_{3} L_{33} \\
L_{21} & L_{22} & l_{3} L_{33} \\
l_{1} L_{21} & l_{2} L_{22} & L_{33}
\end{array}\right],
$$


where

$$
l_{\alpha}=\frac{d^{(3)}\left(p_{\alpha}\right)}{d^{(2)}\left(p_{\alpha}\right)} \quad(\alpha=1,2) \quad l_{3}=\frac{d^{(3)}\left(p_{3}\right)}{d^{(4)}\left(p_{3}\right)},
$$

is obtained from the compatibility equations. The subsequent integration of the strain components, to get the displacement components, introduces the matrix

$$
\mathbf{A}=\left[\mathbf{a}_{1}, \mathbf{a}_{2}, \mathbf{a}_{3}\right]
$$

with

$$
\mathbf{a}_{\alpha}=\left\{\begin{array}{c}
A_{1 \alpha} \\
A_{2 \alpha} \\
A_{3 \alpha}
\end{array}\right\}=\left[\begin{array}{ccc}
s_{16}-s_{11} p_{\alpha}, & s_{12}, & s_{14}-s_{15} p_{\alpha} \\
\frac{s_{26}-s_{21} p_{\alpha}}{p_{\alpha}}, & \frac{s_{22}}{p_{\alpha}}, & \frac{s_{24}-s_{25} p_{\alpha}}{p_{\alpha}} \\
s_{56}-s_{51} p_{\alpha}, & s_{52}, & s_{54}-s_{55} p_{\alpha}
\end{array}\right]\left\{\begin{array}{l}
L_{1 \alpha} \\
L_{2 \alpha} \\
L_{3 \alpha}
\end{array}\right\} .
$$

Eshelby et al. [9] have assumed the displacement vector of the form $\mathbf{u}=$ $\mathbf{a} f\left(x_{1}+p x_{2}\right)$, where $\mathbf{a}=\left\{A_{1}, A_{2}, A_{3}\right\}^{T}$, which is substituted in the equilibrium equation (1) written in terms of the displacement. This leads to another sixthorder polynomial characteristic equation. These two approaches are equivalent. For the BEM formulation Denda [6] and Denda and Marante [7] have adopted Lekhnitskii's approach which gives the explicit expression for the elements of the $\mathbf{A}$ matrix in terms of those of $\mathbf{L}$ matrix. Notice that $\mathbf{L}$ and $\mathbf{A}$ given above include the case when the in-plane and out-of-plane deformations are decoupled. In this case $\left(s_{14}=s_{15}=s_{24}=s_{25}=s_{46}=s_{56}=0\right)$ we have

$$
\mathbf{L}=\left[\begin{array}{ccc}
-p_{1} L_{21} & -p_{2} L_{22} & 0 \\
L_{21} & L_{22} & 0 \\
0 & 0 & L_{33}
\end{array}\right]
$$

and

$\mathbf{A}=\left[\begin{array}{ccc}\left(s_{11} p_{1}{ }^{2}-p_{1} s_{16}+s_{12}\right) L_{21} & \left(s_{11} p_{2}{ }^{2}-p_{2} s_{16}+s_{12}\right) L_{22} & 0 \\ \left(s_{21} p_{1}-s_{26}+\frac{s_{22}}{p_{1}}\right) L_{21} & \left(s_{21} p_{2}-s_{26}+\frac{s_{22}}{p_{2}}\right) L_{22} & 0 \\ 0 & 0 & \left(s_{54}-s_{55} p_{3}\right) L_{33}\end{array}\right]$

Note that for each characteristic root $p_{\alpha}$ we can determine vectors $\mathbf{l}_{\alpha}$ and $\mathbf{a}_{\alpha}$ up to an arbitrary multiplying factor. The solution may be normalized by setting

$$
L_{21}=L_{22}=L_{33}=1
$$

in (8) and (12). The alternative normalization, adopted in this paper, is based on the relations $[1,2]$

$$
\begin{aligned}
\mathbf{L}^{T} \mathbf{A}+\mathbf{A}^{T} \mathbf{L} & =\left[\begin{array}{ccc}
2 \sum_{i=1}^{3} L_{i 1} A_{i 1} & 0 & 0 \\
0 & 2 \sum_{i=1}^{3} L_{i 2} A_{i 2} & 0 \\
0 & 0 & 2 \sum_{i=1}^{3} L_{i 3} A_{i 3}
\end{array}\right], \\
\mathbf{L}^{T} \overline{\mathbf{A}}+\mathbf{A}^{T} \overline{\mathbf{L}} & =\mathbf{0}
\end{aligned}
$$


where $\mathbf{0}$ is the $3 \times 3$ zero matrix and a bar and the superscript $T$ indicate the complex conjugate and the transpose, respectively. The normalization is introduced by setting

$$
2 \sum_{i=1}^{3} L_{i \alpha} A_{i \alpha}=1 \quad(\alpha=1,2,3) .
$$

This gives the orthogonality relations

$$
\begin{aligned}
& \mathbf{L}^{T} \mathbf{A}+\mathbf{A}^{T} \mathbf{L}=\mathbf{I}=\overline{\mathbf{L}}^{T} \overline{\mathbf{A}}+\overline{\mathbf{A}}^{T} \overline{\mathbf{L}} \\
& \mathbf{L}^{T} \overline{\mathbf{A}}+\mathbf{A}^{T} \overline{\mathbf{L}}=\mathbf{0}=\overline{\mathbf{L}}^{T} \mathbf{A}+\overline{\mathbf{A}}^{T} \mathbf{L}
\end{aligned}
$$

where $\mathbf{I}$ is the $3 \times 3$ identity matrix. A through investigation on these matrices, $A_{i \alpha}$ and $L_{i \alpha}$, has been given by Stroh [1, 2], Ting [3], Suo [4] and Ni and Nemat-Nasser [5].

Using $A_{i \alpha}$ and $L_{i \alpha}$ we can represent the displacement $u_{i}$, stress $\sigma_{i j}$ and the stress function $\phi_{i}$ in the form

$$
\begin{aligned}
u_{i}(z) & =2 \Re\left[\sum_{\alpha=1}^{3} A_{i \alpha} f_{\alpha}\left(z_{\alpha}\right)\right], & & \phi_{i}(z)=2 \Re\left[\sum_{\alpha}^{3} L_{i \alpha} f_{\alpha}\left(z_{\alpha}\right)\right] \\
\sigma_{2 i}(z) & =2 \Re\left[\sum_{\alpha=1}^{3} L_{i \alpha} f^{\prime}{ }_{\alpha}\left(z_{\alpha}\right)\right], & & \sigma_{1 i}(z)=-2 \Re\left[\sum_{\alpha=1}^{3} L_{i \alpha} p_{\alpha} f^{\prime}{ }_{\alpha}\left(z_{\alpha}\right)\right],
\end{aligned}
$$

where ( )' indicates the derivative with respect to the argument of the function and $\Re$ is the real part of a complex variable.

\section{Fundamental Solutions}

\subsection{Derivation}

Consider a point force and a dislocation of magnitudes $\mathbf{r}=\left\{r_{1}, r_{2}, r_{3}\right\}^{T}$ and $\mathbf{b}=$ $\left\{b_{1}, b_{2}, b_{3}\right\}^{T}$, respectively located at $\xi=\eta_{1}+i \eta_{2}$ in the $z$-plane and introduce an arbitrary circuit $\Gamma$ around $\xi$, which induces an additional circuit $\Gamma_{\alpha}$ in each of the $z_{\alpha}$-plane as shown in Figure 1. We determine the complex potential function vector $\left\{f_{1}\left(z_{1}\right), f_{2}\left(z_{2}\right), f_{3}\left(z_{3}\right)\right\}^{T}$, such that the force resultant and the displacement jump around the circuit $\Gamma$ are $-\mathbf{r}$ and $\mathbf{b}$, respectively. First notice that the resultant force is obtained by integrating the traction $t_{k}$ around the circuit to get

$$
\begin{aligned}
r_{k} & =\int_{\Gamma} t_{k} d s=\int_{\Gamma} \sigma_{j k} n_{j} d s=\int_{\Gamma}\left(\sigma_{1 k} n_{1}+\sigma_{2 k} n_{2}\right) d s \\
& =\int_{\Gamma}\left\{\left(\frac{\partial \phi_{k}}{\partial x_{2}}\right) \frac{d x_{2}}{d s}+\left(\frac{\partial \phi_{k}}{\partial x_{1}}\right) \frac{d x_{1}}{d s}\right\} d s \\
& =-\left[\phi_{k}\right]_{I}^{F}
\end{aligned}
$$



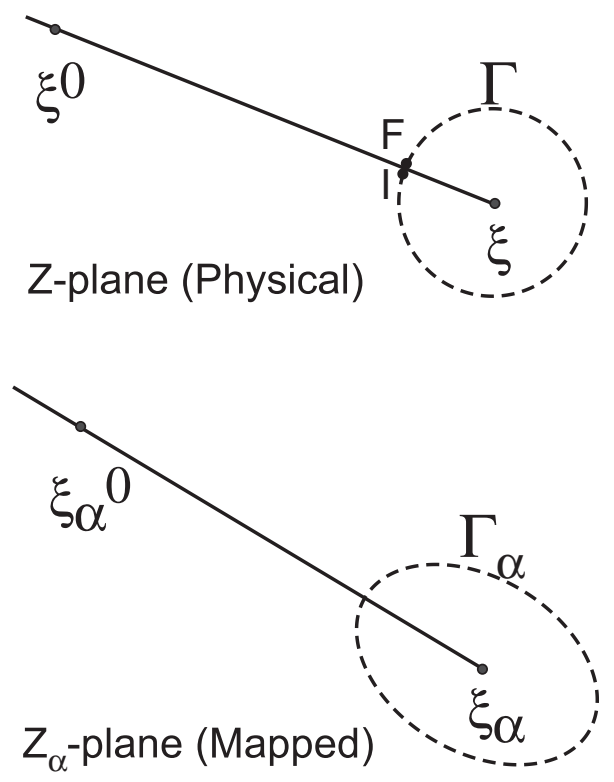

Figure 1: Physical $(z)$ and mapped $\left(z_{\alpha} ; \alpha=1,2,3\right)$ planes. The source point $\xi$ is mapped to $\xi_{\alpha}$. Images $\xi_{\alpha}^{0}(\alpha=1,2,3)$ of the branch line point $\xi^{0}$ are used to define the branch cuts of $\log \left(z_{\alpha}-\xi_{\alpha}\right)$ in the mapped planes.

where the circuit starts at $I$ and ends at $F=I$. Since the logarithmic function is the only possible function that satisfies the force resultant and displacement jump along the prescribed circuit $\Gamma_{\alpha}$ in the $z_{\alpha}$-plane, let

$$
f_{\alpha}\left(z_{\alpha}\right)=\frac{C_{\alpha}}{2 \pi i} \ln \left(z_{\alpha}-\xi_{\alpha}\right) \quad(\alpha=1,2,3),
$$

where $\xi_{\alpha}=\eta_{1}+p_{\alpha} \eta_{2}$ and $C_{\alpha}$ is determined below. Note that there is no sum on $\alpha$. Substituting the complex potential function vector (20) into the displacement and stress function equations (18) we arrive at,

$$
\begin{aligned}
\phi_{k} & =2 \Re \sum_{\alpha=1}^{3} L_{k \alpha} \frac{C_{\alpha}}{2 \pi i} \ln \left(z_{\alpha}-\xi_{\alpha}\right), \\
u_{k} & =2 \Re \sum_{\alpha=1}^{3} A_{k \alpha} \frac{C_{\alpha}}{2 \pi i} \ln \left(z_{\alpha}-\xi_{\alpha}\right) .
\end{aligned}
$$

Calculate the force resultant and the displacement jump around the circuit $\Gamma$ and set them to $-\mathbf{r}$ and $\mathbf{b}$, respectively, to get

$$
-r_{k}=-2 \Re\left[\sum_{\alpha=1}^{3} L_{k \alpha} f_{\alpha}\left(z_{\alpha}\right)\right]_{I}^{F}=-2 \Re\left[\sum_{\alpha=1}^{3} L_{k \alpha} C_{\alpha}\right]=-\sum_{\alpha=1}^{3}\left[L_{k \alpha} C_{\alpha}+\bar{L}_{k \alpha} \bar{C}_{\alpha}\right],
$$




$$
b_{k}=-2 \Re\left[\sum_{\alpha=1}^{3} A_{k \alpha} f_{\alpha}\left(z_{\alpha}\right)\right]_{I}^{F}=2 \Re\left[\sum_{\alpha=1}^{3} A_{k \alpha} C_{\alpha}\right]=\sum_{\alpha=1}^{3}\left[L_{k \alpha} C_{\alpha}+\bar{L}_{k \alpha} \bar{C}_{\alpha}\right],
$$

the component for of which is given by

$$
\left[\begin{array}{llllll}
A_{11} & A_{12} & A_{13} & \bar{A}_{11} & \bar{A}_{12} & \bar{A}_{13} \\
A_{21} & A_{22} & A_{23} & \bar{A}_{21} & \bar{A}_{22} & \bar{A}_{23} \\
A_{31} & A_{32} & A_{33} & \bar{A}_{31} & \bar{A}_{32} & \bar{A}_{33} \\
L_{11} & L_{12} & L_{13} & \bar{L}_{11} & \bar{L}_{12} & \bar{L}_{13} \\
L_{21} & L_{22} & L_{23} & \bar{L}_{21} & \bar{L}_{22} & \bar{L}_{23} \\
L_{31} & L_{32} & L_{33} & \bar{L}_{31} & \bar{L}_{32} & \bar{L}_{33}
\end{array}\right]\left\{\begin{array}{l}
C_{1} \\
C_{2} \\
C_{3} \\
\bar{C}_{1} \\
\bar{C}_{2} \\
\bar{C}_{3}
\end{array}\right\}=\left\{\begin{array}{c}
b_{1} \\
b_{2} \\
b_{3} \\
r_{1} \\
r_{2} \\
r_{3}
\end{array}\right\}
$$

Pre-multiplying the left and right sides of equation (23) by the matrix shown below to get

$$
\left[\begin{array}{ll}
\mathbf{L}^{\mathbf{T}} & \mathbf{A}^{\mathbf{T}} \\
\overline{\mathbf{L}}^{\mathbf{T}} & \overline{\mathbf{A}}^{\mathbf{T}}
\end{array}\right]\left[\begin{array}{ll}
\mathbf{A} & \overline{\mathbf{A}} \\
\mathbf{L} & \overline{\mathbf{L}}
\end{array}\right]\left\{\begin{array}{l}
\mathbf{C} \\
\overline{\mathbf{C}}
\end{array}\right\}=\left[\begin{array}{ll}
\mathbf{L}^{\mathbf{T}} & \mathbf{A}^{\mathbf{T}} \\
\overline{\mathbf{L}}^{\mathbf{T}} & \overline{\mathbf{A}}^{\mathbf{T}}
\end{array}\right]\left\{\begin{array}{l}
\mathbf{b} \\
\mathbf{r}
\end{array}\right\}
$$

the expansion of which gives

$$
\left[\begin{array}{ll}
\mathbf{L}^{\mathbf{T}} \mathbf{A}+\mathbf{A}^{\mathbf{T}} \mathbf{L} & \mathbf{L}^{\mathbf{T}} \overline{\mathbf{A}}+\mathbf{A}^{\mathbf{T}} \overline{\mathbf{L}} \\
\overline{\mathbf{L}}^{\mathbf{T}} \mathbf{A}+\overline{\mathbf{A}}^{\mathbf{T}} \mathbf{L} & \overline{\mathbf{L}}^{\mathbf{T}} \overline{\mathbf{A}}+\overline{\mathbf{A}}^{\mathbf{T}} \overline{\mathbf{L}}
\end{array}\right]\left\{\begin{array}{l}
\mathbf{C} \\
\overline{\mathbf{C}}
\end{array}\right\}=\left\{\begin{array}{l}
\mathbf{L}^{\mathbf{T}} \mathbf{b}+\mathbf{A}^{\mathbf{T}} \mathbf{r} \\
\overline{\mathbf{L}}^{\mathbf{T}} \mathbf{b}+\overline{\mathbf{A}}^{\mathbf{T}} \mathbf{r}
\end{array}\right\} .
$$

Since the matrix in the left hand side of (25) is a unit matrix, due to the orthogonality relations (17), this equation can be readily solved to get

$$
C_{\alpha}=\sum_{k=1}^{3}\left(L_{k \alpha} b_{k}+A_{k \alpha} r_{k}\right) \quad(\alpha=1,2,3),
$$

which can be substituted into (20) to obtain

$$
f_{\alpha}\left(z_{\alpha}\right)=\frac{1}{2 \pi i} \ln \left(z_{\alpha}-\xi_{\alpha}\right) \sum_{k=1}^{3}\left(L_{k \alpha} b_{k}+A_{k \alpha} r_{k}\right) \quad(\alpha=1,2,3) .
$$

It should be noted that there is no sum on $\alpha$.

The displacement and stress contributions due to the line force and line dislocation are given by substituting (27) into (18) to get

$$
u_{i}(z)=\Re\left\{\frac{1}{\pi i} \sum_{\alpha=1}^{3} A_{i \alpha} \ln \left(z_{\alpha}-\xi_{\alpha}\right) \sum_{k=1}^{3}\left(L_{k \alpha} b_{k}+A_{k \alpha} r_{k}\right)\right\}
$$

and

$$
\begin{aligned}
\sigma_{1 i}(z) & =-\Re\left\{\frac{1}{\pi i} \sum_{\alpha=1}^{3} p_{\alpha} L_{i \alpha} \frac{1}{z_{\alpha}-\xi_{\alpha}} \sum_{k=1}^{3}\left(L_{k \alpha} b_{k}+A_{k \alpha} r_{k}\right)\right\} \\
\sigma_{2 i}(z) & =\Re\left\{\frac{1}{\pi i} \sum_{\alpha=1}^{3} L_{i \alpha} \frac{1}{z_{\alpha}-\xi_{\alpha}} \sum_{k=1}^{3}\left(L_{k \alpha} b_{k}+A_{k \alpha} r_{k}\right)\right\} .
\end{aligned}
$$


The traction contribution is calculated by the stress formulas in (29) and the relation $t_{i}=\sigma_{j i} n_{j}$ to get

$$
t_{i}(z)=\Re\left\{\frac{1}{\pi i} \sum_{\alpha=1}^{3}\left(-p_{\alpha} n_{1}+n_{2}\right) L_{i \alpha} \frac{1}{z_{\alpha}-\xi_{\alpha}} \sum_{k=1}^{3}\left(L_{k \alpha} b_{k}+A_{k \alpha} r_{k}\right)\right\} .
$$

\subsection{Explicit Formulas}

\subsubsection{Line Force}

Consider a line force in $x_{k}$ direction applied at $\left(\eta_{1}, \eta_{2}\right)$. The the displacement component in the $x_{i}$ direction at $\left(x_{1}, x_{2}\right)$, denoted by $G_{i k}\left(x_{1}, x_{2} ; \eta_{1}, \eta_{2}\right)$, is given from (28) by

$$
G_{i k}\left(x_{1}, x_{2} ; \eta_{1}, \eta_{2}\right)=\Im \frac{1}{\pi} \sum_{\alpha=1}^{3} A_{i \alpha} A_{k \alpha} \ln \left(z_{\alpha}-\xi_{\alpha}\right),
$$

where $\Im$ indicates the imaginary part. The traction component in the $x_{i}$ direction at $\left(x_{1}, x_{2}\right)$, denoted by $H_{i k}\left(x_{1}, x_{2} ; \eta_{1}, \eta_{2}\right)$, is given from (30) by

$$
H_{i k}\left(x_{1}, x_{2} ; \eta_{1}, \eta_{2}\right)=\Im \frac{1}{\pi} \sum_{\alpha=1}^{3}\left(-p_{\alpha} n_{1}+n_{2}\right) L_{i \alpha} A_{k \alpha} \frac{1}{z_{\alpha}-\xi_{\alpha}} .
$$

The corresponding stress component $\sigma_{i j}$ at $\left(x_{1}, x_{2}\right)$ is denoted by $S_{i j k}\left(x_{1}, x_{2} ; \eta_{1}, \eta_{2}\right)$ and given, from (29), by

$$
\begin{aligned}
& S_{1 i k}(z)=-\frac{1}{\pi} \Im\left\{\sum_{\alpha=1}^{3} p_{\alpha} L_{i \alpha} A_{k \alpha} \frac{1}{z_{\alpha}-\xi_{\alpha}}\right\}, \\
& S_{2 i k}(z)=\frac{1}{\pi} \Im\left\{\sum_{\alpha=1}^{3} L_{i \alpha} A_{k \alpha} \frac{1}{z_{\alpha}-\xi_{\alpha}}\right\} .
\end{aligned}
$$

\subsubsection{Line Dislocation}

Consider a line dislocation at $\left(\eta_{1}, \eta_{2}\right)$ with the unit Burgers vector in $x_{k}$ direction. The resulting displacement component in the $x_{i}$ direction at $\left(x_{1}, x_{2}\right)$, denoted by $P_{i k}\left(x_{1}, x_{2} ; \eta_{1}, \eta_{2}\right)$, is given from (28) by

$$
P_{i k}\left(x_{1}, x_{2} ; \eta_{1}, \eta_{2}\right)=\Im \frac{1}{\pi} \sum_{\alpha=1}^{3} A_{i \alpha} L_{k \alpha} \ln \left(z_{\alpha}-\xi_{\alpha}\right) .
$$

The traction component in the $x_{i}$ direction at $\left(x_{1}, x_{2}\right)$, denoted by $Q_{i k}\left(x_{1}, x_{2} ; \eta_{1}, \eta_{2}\right)$, is given from (30) by

$$
Q_{i k}\left(x_{1}, x_{2} ; \eta_{1}, \eta_{2}\right)=\Im \frac{1}{\pi} \sum_{\alpha=1}^{3}\left(-p_{\alpha} n_{1}+n_{2}\right) L_{i \alpha} L_{k \alpha} \frac{1}{z_{\alpha}-\xi_{\alpha}} .
$$


The corresponding stress component $\sigma_{i j}$ at $\left(x_{1}, x_{2}\right)$ is denoted by $T_{i j k}\left(x_{1}, x_{2} ; \eta_{1}, \eta_{2}\right)$ and given, from (29), by

$$
\begin{aligned}
& T_{1 i k}\left(x_{1}, x_{2} ; \eta_{1}, \eta_{2}\right)=-\frac{1}{\pi} \Im\left\{\sum_{\alpha=1}^{3} p_{\alpha} L_{i \alpha} L_{k \alpha} \frac{1}{z_{\alpha}-\xi_{\alpha}}\right\} \\
& T_{2 i k}\left(x_{1}, x_{2} ; \eta_{1}, \eta_{2}\right)=\frac{1}{\pi} \Im\left\{\sum_{\alpha=1}^{3} L_{i \alpha} L_{k \alpha} \frac{1}{z_{\alpha}-\xi_{\alpha}}\right\} .
\end{aligned}
$$

\section{Computer Codes}

Variables used by the line force and dislocation are listed in Table 1. Subroutines for the line force and line dislocation are listed in Tables 2 and 3. FORTRAN 90 codes and sample input/output files accompany this paper.

\subsection{Normalization used}

A dimensional quantity $q$ is normalized by its reference value $q_{0}$ to define its normalization $\tilde{q}=q / q_{0}$. We select the reference values for the stress and strain to be $\sigma_{0}=10^{8}\left(\mathrm{~N} / \mathrm{m}^{2}\right)$ and $\epsilon_{0}=10^{-3}$. Other reference values are determined such that the normalized governing equations remain exactly the same form as the original equations. These are $s_{0}=10^{-11}\left(\mathrm{~m}^{2} / N\right)$ for the compliance and $u_{0}=\epsilon_{0} x_{0}(m)$ for the displacement, where $x_{0}$ is the characteristic length of the problem. All variables in the codes are normalized and they have magnitudes of order one. To recover the dimensional quantities just multiply the numerical results by the reference values. For example, $G_{i k}=u_{0} \tilde{G}_{i k}\left(P_{i k}=u_{0} \tilde{P}_{i k}\right), H_{i k}=\sigma_{0} \tilde{H}_{i k}$ $\left(Q_{i k}=\sigma_{0} \bar{Q}_{i k}\right)$ and $S_{\text {oik }}=\sigma_{0} \tilde{S}_{\text {oik }}\left(T_{\text {oik }}=\sigma_{0} \tilde{T}_{\text {oik }}\right)$, where quantities with tildes $\left(^{\sim}\right)$ are numerical values obtained by the codes.

\subsection{Branch of logarithmic functions}

For the line dislocation, the branch line of $\ln (z-\xi)$ (in the physical plane) coincides with the slip line, which is assumed to be straight. Given the dislocation at the source point $\xi$ (in the physical plane), the straight slip line is defined by specifying another arbitrary point, $\xi^{0}$ (called the branch line point), on this line as shown in Figure 1. The slip line is given by a line emanating from $\xi$ and extending to and past $\xi^{0}$. The logarithmic function $\ln \left(z_{\alpha}-\xi_{\alpha}\right)$ in each of the mapped $z_{\alpha}$-plane $(\alpha=1,2,3)$ is defined as follows. First, get the image, $\xi_{\alpha}^{0}$, of the branch line point $\xi^{0}$ (Figure 1). Next, calculate the principal value argument of $\xi_{\alpha}^{0}-\xi_{\alpha}$, which is a line from the source $\xi_{\alpha}$ to the branch line point $\xi_{\alpha}^{0}$ in the mapped $z_{\alpha}$-plane. Set this value as the maximum, upang(ia), of the angular range so that

$$
\text { upang(ia) }-\pi<\arg \left(z_{\alpha}-\xi_{\alpha}\right) \leq \operatorname{upang}(\mathrm{ia}) \text {, }
$$


where the minimum is given by upang(ia) $-\pi$. For the line force we use the principal value of the logarithm so that upang(ia) is always set to $\pi$ and it is not necessary to specify $\xi^{0}$.

\subsection{Tips}

- For materials, such as isotropic solids, the characteristic roots are not distinct. Modify the compliance coefficients slightly to make these roots distinct.

- Use the compliance with the original dimension. The code will divide them by $10^{-11} \mathrm{~m}^{2} / N$ for normalization so that the actual values used in computation is of the order of one.

- Subroutine CHARACEQN uses MSIMSL (IMSL for COMPAC FORTRAN) to get characteristic roots of polynomial equations. Use other polynomial roots solver subroutine if MSIMSL is not available and please send the subroutine to the author (denda@jove.rutgers.edu) for future revision.

\section{References}

[1] A. N. Stroh. Dislocations and cracks in anisotropic elasticity. Phil. Mag., Vol. 7:pp. 625-646, 1958.

[2] A. N. Stroh. Steady state problems in anisotropic elasticity. J. Math. Phys., Vol. 41:pp. 77-103, 1962.

[3] T. C. T. Ting. Anisotropic Elasticity: Theory and Applications. Oxford University Press, New York, 1996.

[4] Z. Suo. Singularities, interfaces and cracks in dissimilar anisotropic media. Proc. R. Soc. Lond., Vol. A 427:pp. 331-358, 1990.

[5] L. Ni and S. Nemat-Nasser. General duality principle in elasticity. Mech. Materials, Vol. 24(2):pp. 87-123, 1996.

[6] M. Denda. Mixed mode I, II and III analysis of multiple cracks in plane anisotropic solids by the BEM: a dislocation and point force approach. Int. J. of Engng. Anal. with Boundary Elements, Vol. 25(4-5):pp. 267-278, 2001.

[7] M. Denda and M.E. Marante. Mixed mode BEM analysis of multiple curvilinear cracks in the general anisotropic solids by the crack tip singular element. Int. J. Solids and Struct., Vol. 41(5-6):pp. 1473-1489, 2004.

[8] S. G. Lekhnitskii. Theory of Elasticity of an Anisotropic Elastic Body. HodenDay, San Francisco, 1963.

[9] J.D. Eshelby, W.T. Read, and W. Shockley. Anisotropic elasticity with applications to dislocation theory. Acta Metall., 1, 1953. 


\begin{tabular}{|c|c|c|c|}
\hline Variable & Type & Dimension & Description \\
\hline $\mathrm{t}$ & real & $(21)$ & 3D compliance \\
\hline $\mathrm{z}$ & real & $(2)$ & observation point \\
\hline $\mathrm{n}$ & real & $(2)$ & unit normal \\
\hline $\mathrm{xi}$ & real & $(2)$ & source point \\
\hline $\mathrm{xi} 0$ & real & $(2)$ & branch cut point $^{*}$ \\
\hline $\mathrm{s}$ & real & $(15)$ & reduced compliance \\
\hline $\mathrm{p}$ & complex & $(3)$ & characteristic roots \\
\hline $\mathrm{a}$ & complex & $(3,3)$ & A matrix \\
\hline $\mathrm{I}$ & complex & $(3,3)$ & L matrix \\
\hline za & complex & $(3)$ & $z_{\alpha}$ \\
\hline $\mathrm{xia}$ & complex & $(3)$ & $\xi_{\alpha}$ \\
\hline upang & real & $(3)$ & upper angles of logarithmic functions \\
\hline $\mathrm{g}$ & real & $(3,3)$ & $G_{i k}\left(P_{i k}\right.$ for line dislocation $)$ \\
\hline $\mathrm{h}$ & real & $(3,3)$ & $H_{i k}\left(Q_{i k}\right.$ for line dislocation) \\
\hline stress & real & $(2,3,3)$ & $S_{o i k}\left(T_{o i k}\right.$ for line dislocation) \\
\hline
\end{tabular}

Table 1: FORTRAN variables used for line force and dislocation. Double precision is used for real and complex. ${ }^{*}$ Line force subroutines do not use xio and upang.

\begin{tabular}{|c|c|c|c|}
\hline \multirow{2}{*}{ Subroutine } & \multicolumn{2}{|c|}{ Argument } & \multirow{2}{*}{ Description } \\
\cline { 2 - 3 } & $\mathrm{In}$ & Out & $\begin{array}{c}\text { Input 3D compliance \& } \\
\text { source/observation points data }\end{array}$ \\
\hline Input & $\mathrm{t}, \mathrm{z}, \mathrm{n}, \mathrm{xi}$ & & Reduced compliance \\
\hline REDCOMPLIANCE & $\mathrm{t}$ & $\mathrm{s}$ & Characteristic roots \\
\hline CHARACEQN & $\mathrm{s}$ & $\mathrm{p}$ & L and A matrices \\
\hline LACOEF & $\mathrm{s}, \mathrm{p}$ & $\mathrm{l}, \mathrm{a}$ & $G_{i k}$ \\
\hline GENCOMPVAR & $\mathrm{z}, \mathrm{xi}, \mathrm{p}$ & $\mathrm{za}, \mathrm{xia}$ & Generalized complex variables \\
\hline GMAT & $\mathrm{za}, \mathrm{xia}, \mathrm{a}, \mathrm{l}$ & $\mathrm{g}$ & $H_{i k}$ \\
\hline HMAT & $\mathrm{za}, \mathrm{n}, \mathrm{xia}, \mathrm{a}, \mathrm{l}, \mathrm{p}$ & $\mathrm{h}$ & $S_{o i k}$ \\
\hline SMAT & $\mathrm{za}, \mathrm{xia}, \mathrm{a}, \mathrm{l}$ & $\mathrm{stress}$ & Write results \\
\hline WriteGHS & & $\mathrm{g}, \mathrm{h}$, stress & \\
\hline
\end{tabular}

Table 2: Subroutines for the line force. 


\begin{tabular}{|c|c|c|c|}
\hline \multirow{2}{*}{ Subroutine } & \multicolumn{2}{|c|}{ Argument } & \multirow{2}{*}{ Description } \\
\cline { 2 - 3 } & $\mathrm{In}$ & Out & $\begin{array}{c}\text { Input 3D compliance \& } \\
\text { source/observation points data }\end{array}$ \\
\hline Input & $\mathrm{t}, \mathrm{z}, \mathrm{n}, \mathrm{xi}, \mathrm{xi} 0$ & & Reduced compliance \\
\hline REDCOMPLIANCE & $\mathrm{t}$ & $\mathrm{s}$ & Characteristic roots \\
\hline CHARACEQN & $\mathrm{s}$ & $\mathrm{p}$ & L and A matrices \\
\hline LACOEF & $\mathrm{s}, \mathrm{p}$ & $\mathrm{l}, \mathrm{a}$ & $P_{i k}$ \\
\hline GENCOMPVAR & $\mathrm{z}, \mathrm{xi}, \mathrm{xi} 0, \mathrm{p}$ & $\mathrm{za}, \mathrm{xia}, \mathrm{upang}$ & Generalized complex variables \\
\hline PMAT & $\mathrm{za}, \mathrm{xia}, \mathrm{upang}, \mathrm{a}, \mathrm{l}$ & $\mathrm{g}$ & $Q_{i k}$ \\
\hline QMAT & $\mathrm{za}, \mathrm{n}, \mathrm{xia}, \mathrm{a}, \mathrm{l}, \mathrm{p}$ & $\mathrm{h}$ & $T_{\text {oik }}$ \\
\hline TMAT & $\mathrm{za}, \mathrm{xia}, \mathrm{a}, \mathrm{l}$ & stress & Write results \\
\hline WriteGHS & & $\mathrm{g}, \mathrm{h}$, stress & \\
\hline
\end{tabular}

Table 3: Subroutines for line dislocation. 